\title{
Oper in der Toskana in der zweiten Hälfte des 18. Jahrhunderts: Zur Verbreitung der Gattung auf dem Lande
}

\author{
von Christine Siegert
}

$-1-$

"In jeder kleinen Stadt, in jedem Dorfe findet man ein Theater errichtet. Bloß allein der paebstliche Staat zaehlt ihrer mehr als vierzig. Es mag den Armen an Unterhalt fehlen, den Fluessen an Bruecken, den Feldern an Abfluessen, den Schwachen an Krankenhaeusern, und an Huelfsmitteln fuer oeffentliche Ungluecksfaelle, an einer Art von Theater fuer die Muessiggaenger wird es gewiß nie fehlen. Die Frage an den, welcher am Ruder der Regierung sitzt, was in unsern Zeiten das italiaenische Volk mache, ist dieselbe, die man vor sechzehn Jahrhunderten, in den Zeiten Juvenals that, und sie wuerde ebenfalls beantwortet werden muessen: Lebensmittel und Schauspiele, Panem et Circenses. In jedem Jahr werden hier mehr als ein halbes hundert verschiedene musikalische Vorstellungen aufgefuehrt."

Dieses Zitat des Operntheoretikers Estebán de Arteaga in der Übersetzung Johann Nikolaus Forkels gibt einen Hinweis auf einen bislang vernachlässigten Rezeptionsstrang des Musiktheaters: die Verbreitung der Oper im dörflichen und kleinstädtischen Bereich in Italien. Die Beschäftigung mit diesem Phänomen eröffnet als Ergänzung bisheriger Untersuchungen neue Perspektiven auf die Geschichte des Musiktheaters, denn das reiche Theaterleben, das im 18. Jahrhundert auch in kleineren Kommunen stattfand, ist von der Musiktheaterwissenschaft bislang noch viel zu wenig zur Kenntnis genommen worden. Sie hat sich auf die - zweifellos für die Gattungsgeschichte wesentliche - geographisch breite Diffusion einzelner Opern in den Metropolen und Residenzen Europas konzentriert. Parallel dazu fand jedoch eine Verbreitung "nach innen" statt, in kleine Ortschaften, was allenfalls in - methodisch häufig zweifelhaften -

\footnotetext{
1 Esteban Arteaga, Geschichte der italiänischen Oper, Aus dem Italiänischen übersetzt und mit Anmerkungen begleitet von Johann Nicolaus Forkel, Nachdr. der Ausg. Leipzig 1789, 2 Teile in einem Bd., Hildesheim, New York 1973, Teil 2, S. 283-284.
} 
regionalgeschichtlichen Studien erwähnt wird. Doch, um es zugespitzt zu formulieren: Eine Aufführung in einer Tausend-Seelen-Gemeinde ist für eine strukturgeschichtliche Forschung nicht weniger bedeutsam als eine Gala-Vorstellung an der Mailänder Scala.

$$
-2-
$$

Es kann nicht oft genug betont werden, wie stark die historische Sicht auf das Musiktheater (und nicht nur auf dieses) von der Quellengrundlage abhängt. So vertritt Reinhard Wiesend die optimistische Auffassung, daß die Spielpläne "durch die erhaltenen Librettodrucke [...] ziemlich genau rekonstruiert werden" ${ }^{2}$ können. Daß dies nur für die Opernzentren und -mittelzentren gilt, zeigt sich schnell, wenn man die zeitgenössische Presse zu Rate zieht, aus der eine bedeutend breitere Distribution der Gattung hervorgeht, ohne daß häufig Spielpläne über eine längere Zeitspanne ermittelt werden können. Was Arteaga für den Kirchenstaat andeutete, soll im folgenden anhand des Opernlebens in der Toskana der zweiten Hälfte des 18. Jahrhunderts exemplifiziert werden.

$$
-3-
$$

Bereits die systematische Durchsicht eines Jahrgangs der Gazzetta Toscana, einer Florentiner Zeitung, die ab $1766^{3}$ erschien, erbringt den Befund einer Reihe von Musiktheateraufführungen, für die keine Librettodrucke bekannt sind. 1776 wird in dieser Zeitung über Aufführungen u. a. in Pisa (La fraschetana), Montepulciano ( La giardiniera), Prato (Pasquale Anfossi: Il geloso in cimento sowie Giovanni Paisiello: L'innocente fortunata) und dem kleinen Ort Torrita (Niccolò Piccinni: I servi astuti) berichtet $^{4}$, die in den Standardnachschlagewerken von Claudio Sartori, Franz Stieger und Alfred Loewenberg nicht nachgewiesen sind ${ }^{5}$. Aus dem Wissen um diese Aufführungen ergibt sich die Frage nach ihrem kulturellen Umfeld, den institutionellen Voraussetzungen sowie dem gespielten Repertoire. Im folgenden soll dieses ansatzweise re-

2 Reinhard Wiesend, Die italienische Oper im 18. Jahrhundert: Hinführung, in: Herbert Schneider, Reinhard Wiesend (Hg.), Die Oper im 18. Jahrhundert, Unter Mitarbeit von Daniel Brandenburg, Michele Calella, Arnold Jacobshagen, Francesca Menchelli-Buttini, Herbert Schneider, Reinhard Strohm und Reinhard Wiesend, (Handbuch der musikalischen Gattungen, hg. v. Siegfried Mauser, Bd. 12) Laaber 2001, S. 15-21, Zit. S. 15, Anm. 2. Im ersten Jahr unter dem Titel Gazzetta Patria.

Gazzetta Toscana [im folgenden abgekürzt GT] 1776, S. 24, 80, 127, 139, 163. Obwohl die GT für La fraschetana und La giardiniera keinen Autor nennt, kann man einen Zusammenhang mit Paisiellos und Anfossis berühmten Opern aufgrund von deren Beliebtheit vermuten.

5 Vgl. Claudio Sartori, I Libretti italiani a stampa dalle origini al 1800. Catalogo analitico con 16 indici, 5 Bde. und 2 Registerbde., Cuneo 1990-1993; Franz Stieger, Opernlexikon. Titelkatalog, 3 Bde., Tutzing 1975; Alfred Loewenberg, Annals of Opera, 2 Bde., 2. Aufl., Genf 1954. 
konstruiert und in seinem historischen Kontext verortet werden. Dieser besteht insbesondere in der Festkultur von Karneval und Patronatsfeiern. Nach den Ausführungen Arteagas zur Situation im Kirchenstaat ist zu erwarten, daß die Ergebnisse auf andere Regionen Italiens und vielleicht sogar darüber hinaus übertragen werden können. Grenzen dürften einer flächendeckenden Opernpraxis vor allem durch die Gesetzgebung entstanden sein, die selbstverständlich in allen italienischen Staaten unterschiedlich war. Da auch die rechtlichen Grundlagen für das Theaterleben in der Toskana entscheidenden Wandlungen unterworfen waren, soll zuvor ein Überblick über die Entwicklung der Theatergesetzgebung gegeben werden. Als Quellen dienen genuin historische: zeitgenössische Presseberichte und Polizeiakten des Florentiner Staatsarchivs. Den Aufführungen in den einzelnen Orten selbst nachzugehen, wäre sicherlich äußerst interessant, hätte den Rahmen dieses Referats allerdings gesprengt ${ }^{6}$.

\section{Zur Theatergesetzgebung}

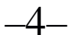

1765 bestieg der achtzehnjährige österreichische Erzherzog Leopold, Sohn Maria Theresias und Franz' I., unter dem Namen Pietro Leopoldo den Thron des Großherzogtums Toskana. Den Idealen der Aufklärung verbunden, brachte der junge Monarch eine Reihe von Reformen in allen Lebensbereichen in Gang: Landwirtschaft, Straßenbau, Handel, Justiz, Religionsausübung, Hygiene und Bildungswesen. Er förderte die Wissenschaften und die Künste, richtete unter anderem das naturhistorische Museum in Florenz ein und gründete die Accademia delle Belle Arti ${ }^{7}$. Das Theater bedeutete für Pietro Leopoldo zunächst vor allem ein Bildungsinstrument. Dementsprechend förderte er den Bau und die Renovierung von Theatern: Der Umbau des Theaters 1779 in Poppi,

6 Der vorliegende Beitrag ist die schriftliche Fassung eines Referats, das auf dem 17. Internationalen Studentischen Symposium des DVSM e. V. Musiktheaterwissenschaft - Eine interdisziplinäre Herausforderung? vom 2. bis 5. Oktober 2002 an der Ruhr-Universität Bochum gehalten wurde. Es ging aus den Arbeiten zu meiner Dissertation Cherubini in Florenz. Studien zur Gattungssituation der Oper in Italien zwischen 1770 und 1790, Hochschule für Musik und Theater Hannover 2003 (masch.) hervor. Die zugrundeliegenden Quellenstudien wurden ermöglicht durch Stipendien des Deutschen Historischen Instituts Rom, des Deutschen Akademischen Austauschdienstes sowie der Gerda Henkel Stiftung. Allen Institutionen möchte ich meinen herzlichen Dank aussprechen.

7 Vgl. Valentino Baldacci (Hg.), Le riforme di Pietro Leopoldo e la nascita della Toscana moderna, Presentazione di Riccardo Nencini e Claudio Martini, Testi di Valentino Baldacci, Franco Cardini, Cosimo Ceccuti, Zeffiro Ciuffoletti, Romano Paolo Coppini, Maurizio Degl'Innocenti, Leonardo Rombai, Aldo Schiavone, Marcello Verga, Firenze 2000. 
einer Gemeinde in der Provinz Arezzo, beruhte beispielsweise auf seiner Anweisung ${ }^{8}$. Als aber die Betreiber des Theaters in Pietrasanta den Großherzog um seine Protektion baten, gewährte der Monarch ihnen ihre Bitte nicht, und zwar, weil er grundsätzlich den Theatern in der Provinz keine Protektion zubilligte. Dennoch darf man sich nicht vorstellen, daß es sich bei diesem Theater nur um einen einfachen Dorfsaal gehandelt hätte. Es verfügte immerhin über drei Ränge mit 37 Logen ${ }^{9}$. Wie stolz eine Gemeinde auf ihr Theater war, kann man ermessen, wenn man einen begeisterten und von Übertreibungen sicherlich nicht ganz freien Bericht der Gazzetta Toscana über das neue Theater in Pontadera zu Rate zieht:

"In unserem neuen Theater, das Sig. Pierantonio Gabbrielli und Söhne konstruiert haben, das Sig. Vincenzio Gabbrielli entworfen hat und das außerordentlich schön geworden ist, sind bisher verschiedene Werke des berühmten und unsterblichen Metastasio aufgeführt worden, und jetzt wird eine Tragödie Abate Ringhieris mit dem Titel Adelasia in Italia aufgeführt. Sonntag Abend den 23. wird das opulente Dramma Olimpiade mit Chören aufgeführt, was der Oper besondere Brillanz verleiht. $\mathrm{Zu}$ diesem Anlaß wird es Gelegenheit geben, das ganze Theater von seiner glänzendsten Seite kennenzulernen, weil das von den Meistern Cipriani und Vater Filippo und Sohn Lenzi neu gestaltete Bühnenbild Verwendung finden wird; der erste unter ihnen ist auf Figuren spezialisiert, der zweite auf Gebäude und Landschaften: Beide haben der Erwartung entsprochen, die ihnen vom Publikum entgegengebracht wurde, indem sie es erfolgreich und meisterhaft im Stil des unsterblichen Raffael gestaltet haben, so daß wir uns rühmen können, nicht nur ein Theater, sondern die schönste Galerie zu besitzen, die man sich vorstellen kann, denn die beeindruckende Ansicht und die abwechslungsreiche Farbgebung, die dieses Werk beseelen und beleben, verdienen echte Bewunderung. "10

$8 \quad$ Vgl. Elvira Garbero Zorzi, Luigi Zangheri, I teatri storici della Toscana. Censimento documentario e architettonico, Bd. IV: Arezzo e Provincia, Firenze 1994, S. 301.

9 Vgl. Archivio di Stato di Firenze [im folgenden abgekürzt I-Fas]: Presidenza del Buongoverno 1784-1808, Affari comuni [im folgenden abgekürzt $P B G$ ] 11, Nr. 818.

10 "In questo nuovo nostro Teatro stato costruito dal Sig. Pierantonio Gabbrielli, e Figli, col disegno del Sig. Vincenzio Gabbrielli, che è riescito uno dei più vaghi che possino mai vedersi, sono state fin quì recitate diverse Opere del celebre, ed immortal Metastasio, e ora si rappresenta una Tragedia dell'Abate Ringhieri intitolata l'Adelasia in Italia. Domenica sera 23. stante sarà posto in Scena il Dramma l'Olimpiade vagamente decorato, con i Cori in musica, lochè renderà l'Opera più brillante. In tale occasione si avrà luogo di vedere tutto il suddetto Teatro nel suo più vago aspetto, giacchè comparirà lo Scenario nuovamente fatto dagli abili Professori Sigg. Cipriano, $e$ Filippo Padre, e Figlio Lenzi, il primo dei quali prescelto per le Figure, e il secondo per l'architettura, e paesi: ambidue hanno corrisposto all'espettativa formata di essi dal Pubblico, 
Die ursprünglich liberale Theaterpolitik, die den Bau von Theatern in der ganzen Toskana förderte, begann einige Jahre vor der beschriebenen Theateröffnung 1783 in Pontadera zum Gegenstand von Kritik zu werden. 1779 erlebte die Hauptstadt Florenz zwei größere Theatergründungen, so daß in der Herbstsaison insgesamt sechs Theater bespielt wurden. Als der Impresario des Teatro di Via Santa Maria um die Genehmigung zur Öffnung in dieser Saison nachsuchte, meldete der Polizeipräsident, obwohl er die Genehmigung befürwortete, Bedenken an:

"Giuseppe Maria del Pace, Impresario des Teatro di Via S[anta] Maria, bittet, daß ihm die Öffnung desselben in der laufenden Saison gestattet werde, um dort von der Schauspieltruppe Ferrari im kommenden Herbst Prosakomödien und -tragödien aufführen zu lassen, nachdem die französischen Vorstellungen beendet sind.

Obwohl in dieser Saison viele Theater geöffnet sind, denke ich, daß Eure Königliche Hoheit auch die Öffnung des Teatro di Via S[anta] Maria genehmigen sollte, umso mehr als Ihr auch in den letzten Jahren diese Gnade gewährt habt $<$ und weil die Zahl der Theater vielleicht die Kraft derjenigen verlangsamen wird, die sie mit Taktlosigkeit befördern;> daher würde ich vorschlagen, zu verfügen: 'Nachdem die übliche Steuer bezahlt ist, wird genehmigt, was beantragt wird'"11.

Als das Teatro della Piazza Vecchia di Santa Maria Novella in derselben Saison die Aufführung von Opere buffe plante, wies der Polizeipräsident erneut auf die große Zahl der Theater hin:

avendo fatta la bravamente, e maestrevolmente sul gusto dell'immortal Raffaello, talchè noi possiamo vantarci di avere non un Teatro, ma bensì la più bella Galleria, che possa immaginarsi, essendo stato colpito il punto di vista e ben messa in uso la varietà dé colori che animano, e ravvivano quest'Opera degna veramente di ammirazione." (GT 1783, S. 31; Übersetzungen hier und im folgenden von der Verf.)

"Giuseppe Maria del Pace Impresario del Teatro di Via S[anta] Maria domanda, che gli sia concessa l'apertura del medesimo nella corrente stagione per rappresentarvi Commedie, e Tragedie in prosa per mezzo della Compagnia Comica Ferrari durante il prossimo futuro autunno, da eminciarsi da principiare dopo che saranno terminate le Commedie Francesi.

Sebbene siano molti i Teatri che in tale Stagione rimarranno aperti, ciò non ostante stimerei, che V[ostra] A[ltezza] R[eale] permettesse l'apertura anche di quello di Via S[anta] Maria, tanto più che anche negli anni passati si è degnata di concedere una simile grazia <e che la moltitudine de' Teatri contribuirà forse a rallentarne il furore in quelli, che gli promovono con indiscretezza;> onde proporrei rescriversi 'Pagata la solita tassa, concedesi come si domanda"" (I-Fas: Camera e auditore fiscale [im folgenden abgekürzt $C A F$ ] 2889, Nr. 1099; bei der Übertragung der Polizeiakten werden Einschübe durch $<>$ gekennzeichnet). 
"Gaspero Mannelli, Impresario des Teatro della Piazza Vecchia di S. M. ${ }^{a}$ Novella, bittet mit beiliegendem Gesuch, dasselbe in der laufenden Saison zu öffnen, um dort Opere buffe in musica aufzuführen.

Die Theater, die bereits geöffnet sind, sind wirklich mehr als genug, um die Einheimischen und dazu die Fremden zu unterhalten; aber weil Eure Königliche Hoheit nichts desto weniger den anderen Impresari die Öffnung der jeweiligen Theater gnädigst gestattet hat, <und weil Eure Königliche Hoheit keinen Befehl und keine Regelung zur Öffnung der Theater ausgesprochen hat, > würde ich denken, daß Ihr geruhen könntet, sie auch diesem Bittsteller zu erlauben; daher würde ich vorschlagen, auf seine Bitten zu verfügen: 'Nachdem die übliche Steuer bezahlt ist, wird genehmigt, was beantragt wird'."12

Zwei Gründe sprechen für die Öffnung des Theaters: Zunächst die Tatsache, daß der Großherzog die Öffnung aller anderen Theater genehmigte und es keinen Grund gibt, warum er sie dem Teatro della Piazza Vecchia di Santa Maria Novella dann verweigern sollte. Zum zweiten habe es der Großherzog versäumt, einen "regolamento per l'apertura de' Teatri" zu erlassen.

\section{$-5-$}

Ende desselben Jahres begannen die Diskussionen darüber, wie eine sinnvolle Regulierung des Theaterangebots aussehen könnte. Als grundsätzliche Argumente für das Theater führte der Polizeipräsident an, daß es Bekannt- und Freundschaften über die Standesgrenzen hinweg ermöglichte und förderte und einen nicht zu vernachlässigenden Wirtschaftsfaktor darstellte, da diese Kontakte den zivilen Handel stimulierten. Außerdem würde fast das gesamte Gehalt des Theaterpersonals - hiermit meinte er wohl vor allem Sängerinnen und Sänger, Tänzerinnen und Tänzer sowie Schauspielerinnen und Schauspieler - in die regionale Wirtschaft zurückfließen, weil die Theaterleute nicht sparen könnten. Als negative Folgen des Theaters für die Moral und die so-

12 "Gaspero Mannelli Impresario del Teatro della Piazza Vecchia di S. M. ${ }^{a}$ Novella domanda coll'annessa Supplica d'aprire il medesimo nella corrente Stagione per rappresentarvi Opere buffe in musica.

Veramente $i$ Teatri, che già sono stati aperti, sono più che sufficienti per divertire $i$ nazionali, ed $i$ Forestieri ancora; Ciò non ostante però siccome V[ostra] A[ltezza] R[eale] ha benignam[ent]e permesso agli altri Impresarj l'apertura dei respettivi Teatri, <V[ostra] A[ltezza] R[eale] non ha per anco spiegato alcun ordine e regolamento per l'apertura de' Teatri; così stimerei, che potesse degnarsi di permetterla anche al supplicante; onde proporrei rescriversi le di lui Preci 'Pagata la consueta Tassa, concedesi come si domanda'." (I-Fas: CAF 2890, Nr. 1132.) 
zialen Bindungen zählte der Polizeipräsident auf, sie brächten selbst angenehme und lehrreiche Gesellschaftsveranstaltungen zum Verschwinden, zerstörten die familiäre Ordnung, kultivierten den Müßiggang und trügen stark zu Verschwendung und Zügellosigkeit bei ${ }^{13}$. Aus diesen Erwägungen folgt für ihn, daß es notwendig sei, "die Freiheit der Theater und Schauspiele damit zu vereinbaren, daß gleichzeitig ihre gefährlichen Konsequenzen verhindert <oder zumindest verringert> werden" ${ }^{14}$. Für die kleineren Ortschaften schlug der Polizeipräsident folgende Regelung vor:

"In den Städten und Gemeinden des Großherzogtums, wo bereits Theaterbauten existieren, würde ich erlauben, daß im Karneval Komödien und Dramen aufgeführt werden; unter der Bedingung jedoch, daß die Akteure Einwohner <des Ortes> sind, <an dem sich das Theater befindet,> oder daß sie zumindest gebürtige Untertanen sind. [...]

Außerhalb des Karnevals würde ich erklären, daß niemandem erlaubt < die Erlaubnis erteilt werden wird,> ohne ausdrückliche Genehmigung Seiner Königlichen Hoheit Schauspielvorstellungen jeder Art zu geben, und diese wird nicht erteilt werden, außer unter der Voraussetzung, daß diese Vorstellungen aus Anlaß von Jahrmärkten und Festen mit Publikumszulauf gegeben und im Karneval nicht wiederholt werden." 15

Der endgültige Erlaß lautete in diesem Punkt folgendermaßen:

"In jeder anderen Stadt [außer Florenz, Siena, Pisa und Livorno, d. Verf.] und in jedem Landsitz oder Ort des Großherzogtums wird die Öffnung eines Theaters nur zur Karnevalszeit erlaubt, und wenn die eine oder andere Stadt oder Gemeinde die Öffnung des Theaters zu einer anderen Zeit aus Anlaß von Jahrmärkten oder Festen wünscht, muß sie frühzeitig um die Erlaubnis nachsuchen, die al-

Vgl. I-Fas: CAF 2893, Nr. 1423.

"[...] di combinare la libertà de' Teatri, e Spettacoli con prevenirne <o diminuirne almeno> le cattive conseguenze" (I-Fas: CAF 2893, Nr. 1423).

15 "Nelle Città, e Terre del Granducato, dove esistono Teatri già costruiti, permetterei, che nel Carnevale si rappresentassero Commedie, ed Opere Drammatiche; a condizione però, che gli Attori fossero Terrazzani <del luogo, dove esiste il Teatro>, o fossero per il meno Sudditi originarj. [...] Fuori del Carnevale dichiarerei, che non sarà permess $\langle a d$ alcuno concessa la licenza $\rangle$ di far Rappresentanze Comiche d'alcuna sorte senza espressa grazia di S[ua] A[ltezza] R[eale], la quale non averà luogo, Se non colla condizione, che facendosi tali Rappresentanze in occasione di Fiere, e Feste di concorso, non debbano rinnuovarsi nel tempo del Carnevale." (I-Fas: CAF 2893, Nr. 1423.) 
lerdings nicht immer und unter allen Umständen erteilt werden wird. Ebensowenig werden Verbindlichkeiten berïcksichtigt, die trotz dieser Bekanntmachung vor einer Genehmigung eingegangen werden." ${ }^{16}$

Unter Pietro Leopoldos Sohn und Nachfolger Ferdinand III. wird für eine derartige Beschränkung folgende Erklärung gegeben, die gleichzeitig einen Hinweis auf die soziale Zusammensetzung des ländlichen Theaterpublikums darstellt: "[I]n diesen Orten gibt es nur wenig Menschen, die ihr Brot nicht mit ihren Händen verdienen müssen; wenn sie abends ins Theater gehen, sind sie am Morgen nicht pünktlich bei der Arbeit"17. Schließlich verbot Pietro Leopoldo 1785 den Theaterbetrieb in den kleineren Orten der Toskana ganz und die Oper in allen Städten bis auf Florenz, Pisa, Siena und Livorno:

"Seine Königliche Hoheit, die der Verschwendung und den schlechten Sitten entgegentreten möchte, die aus dem übermäßigen Theaterbesuch besonders auf dem Land entstehen, gebietet, daß in Zukunft die vorliegende Regelung dauerhaft beachtet wird. I. Der Gebrauch von jeder Art von Masken wird zu jeder Zeit und in jeder Stadt, jedem Dorf, Landsitz und an jedem anderen Ort des Großherzogtums außerhalb der vier Städte Florenz, Siena, Pisa und Livorno vollkommen abgeschafft und für immer verboten. [...] IV. Die Theater in Florenz werden auf nur vier festgesetzt, und zwar die der Via della Pergola, della Palla a Corda bzw. degl'Intrepidi, di Via del Cocomero und di Via S[anta] Maria; und in Siena, Pisa und Livorno wie auch in Pistoja, Arezzo, Volterra, Cortona, Montepulciano, Prato, Pescia, Pontremoli, Colle, Empoli und Pietrasanta darf nur ein einziges Theater für jede dieser Städte und Gemeinden bestehen. V. Alle anderen Theater, die über die oben erwähnten hinaus derzeit sowohl in Florenz als auch in den übrigen Städten, Gemeinden und Ortschaften des Großherzogtums existieren,

16 "In ogni altra Città [außer Florenz, Siena, Pisa und Livorno, d. Verf.], ed in qualunque Castello, o Terra del Gran-Ducato si permetterà l'apertura di un Teatro nel solo tempo di Carnevale, e qualora da alcuna Città, e Terra si volesse l'apertura del Teatro in altro tempo in occasione di Fiere, o di Feste dovrà domandarsene la permissione per tempo, mentre non sempre, ed in tutte le circostanze verrà accordata, ne si avrà riguardo agli impegni che non ostante il presente avviso fossero stati contratti prima di ottenerla." (GT 1779, S. 197.)

17 "[P]oche sono le Persone dei detti luoghi che non abbiano bisogno di guadagnarsi il pane con le loro braccia; se passano la sera al Teatro, non saranno la mattina di buon ora al Lavoro" (I-Fas: Segreteria di Stato 1765-1808, 652, prot. n. 3, 1796: osservazioni del presidente del Buongoverno per la stesura della circolare sui teatri. Zit. nach Loredana Maccabruni, L'Accademia degli Immobili e il Teatro della Pergola dai sovrani lorenesi al regno d'Italia, in: Marcello de Angelis, Elvira Garbero Zorzi, Loredana Maccabruni, Piero Marchi, Luigi Zangheri (Hg.), Lo "spettacolo maraviglioso". Il Teatro della Pergola: l'opera a Firenze, Archivio di Stato di Firenze 6 ottobre 30 dicembre 2000, Firenze 2000, S. 47-59, Zit. S. 53). 
werden für immer abgeschafft. Zu diesem Zweck wird den Besitzern derselben die Frist von zehn Monaten vom Tag der Veröffentlichung dieses Dekrets angezeigt, innerhalb derer sie sie abgerissen, umgebaut oder einer anderen Bestimmung zugeführt haben müssen; und die Quartierskommissare in Florenz und die Königlichen Landvögte in der Provinz werden beauftragt, die Umsetzung dieses Befehls zu überwachen, um der Regierung jene anzuzeigen, die nach der vorgeschriebenen Frist möglicherweise noch existieren." 18

Auch die Spielzeiten und das Repertoire wurden festgeschrieben:

"VI. In den vier Städten Florenz, Siena, Pisa und Livorno dürfen die Theater in der Frühjahrs-, Herbst- und Karnevalssaison öffnen; und in den anderen obengenannten Städten und Ortschaften dürfen sie nur in der Karnevalszeit öffnen; jedoch mit der Auflage, daß auch in Florenz in der Frühjahrs- und Herbstsaison nie mehr als zwei gleichzeitig in derselben Saison geöffnet sein dürfen. VII. Nur in den Theatern von Florenz, Siena, Pisa und Livorno dürfen Opere serie und Burlette in musica mit Ballett aufgeführt werden; aber außer ihnen werden in keinem weiteren Theater musikalische Vorstellungen oder Ballette jeglicher Art erlaubt werden, denn es dürfen nur Komödien und Tragödien aufgeführt werden. VIII. Auch alle szenischen Aufführungen in Privathäusern unter welchem Namen oder Vorwand auch immer, auch zum reinen Vergnügen der betreffenden einzelnen Familien werden in Zukunft verboten, wenn sie gegen Eintritt, Einladung und Eintrittskarten veranstaltet werden, weil Seine Königliche Hoheit gebietet, daß derartige Aufführungen wie oben beschrieben nur in den Theatern stattfinden sollen, die oben erlaubt werden. Ausdrücklich werden alle Theater oder Theater-

"S[ua] A[ltezza] R[eale] Volendo rimediare alla dissipazione, e mal costume, che derivano dalla troppo frequenza de' Teatri, specialmente nelle Campagne, Vuole, che in avvenire stabilmente si osservi il presente Regolamento. I. Sarà onninamente abolito, e proibito per sempre l'uso delle Maschere di qualunque sorte, in qualunque tempo, ed in qualunque Città, Terra, Castello, e altro luogo del Granducato, fuori che nelle quattro Città di Firenze, Siena, Pisa, e Livorno. [...] IV. I Teatri in Firenze restano fissati a soli quattro; quelli cioè di Via della Pergola, della Palla a Corda o sia degl'Intrepidi, di Via del Cocomero, e di Via S[anta] Maria; Ed in Siena, Pisa, e Livorno, come pure in Pistoja, Arezzo, Volterra, Cortona, Montepulciano, Prato, Pescia, Pontremoli, Colle, Empoli, e Pietrasanta non dovrà esistere se non un solo Teatro per ciascheduna di d[ette] Città e Terre. V. Tutti gli altri Teatri che attualmente esistono tanto in Firenze, che nelle altre Città, Terre, e Luoghi del Granducato, fuori di quelli sopra indicati restano per sempre aboliti. A questo oggetto viene assegnato ai Proprietarj dei medesimi il termine di dieci Mesi da decorrere dal dì della pubblicazione di questo Regolamento ad averli disfatti, alienati, o ridotti ad altri usi; E i Commissari de' Quartieri in Firenze, ed $i$ Vicarj Regj in Provincia restano incaricati d'invigilare all'esecuzione di quest'Ordine per rendere conto al Governo di quelli che dopo il tempo prescritto vi fossero ancora esistenti." (GT 1785, S. 61.) 
vorstellungen in den Mönchs- und Nonnenklöstern, Konservatorien und Bildungskollegien jeder Art verboten, auch wenn sie unter Titeln wie Pastorali sacre etc. laufen; und wo entsprechende Theater existieren, müssen dieselben sofort abgerissen werden." 19

Schließlich regelte der Erlaß auch die Sonderveranstaltungen:

"IX. In Florenz werden im Teatro di Via della Pergola die üblichen Faschingsbälle am letzten Donnerstag-, Sonntag- und Dienstagabend des Karnevals gestattet; und im Teatro degli Intrepidi, bzw. della Palla a Corda an den beiden letzten Montagabenden und am vorletzten Sonntagabend des Karnevals. In allen anderen Städten und Dörfern, in denen der Brauch der Faschingsbälle erlaubt ist, am letzten Donnerstag-, Sonntag-, Montag- und Dienstagabend des Karnevals. $X$. Unterschiedslos werden schließlich alle Vorstellungen, die von den Impresari oder anderen entweder aufgrund von Vereinbarungen oder aus anderem Grund dem Gewinn und Vorteil der Schauspieler, Sänger oder Tänzer oder einzelnen unter ihnen vorbehalten wurden und gewöhnlich Benefizvorstellungen genannt werden, in Zukunft eingestellt und abgeschafft und unter keinem Namen oder Vorwand erlaubt. XI. Alle anderen Faschingsveranstaltungen und Bälle gegen Eintritt oder Einladung in Privathäusern werden generell verboten, wenn nicht vorher in Florenz von den Quartierskommissaren und in der Provinz von den jeweiligen Richtern die Erlaubnis eingeholt wird; wohlverstanden jedoch, daß eine solche Erlaubnis nur Personen von erwiesener Rechtschaffenheit und Umsicht erteilt wird, und zwar mit der Auflage, daß das Familienoberhaupt für alle Un- 
annehmlichkeiten haftet, die es bei größerer Aufmerksamkeit hätte verhindern können. ${ }^{\prime 20}$

Selbstverständlich erließ der Monarch all diese Regelungen nur zum Wohl seiner Untertanen:

"Seine Königliche Hoheit, die immer darauf bedacht ist, die guten Sitten zu fördern und jene Mittel auszurotten, die das Laster schüren, befiehlt und gebietet, daß in Zukunft die vorliegende Regelung ständig beachtet wird, denn sie hat erkannt, von welchem Schaden das Vorhandensein von Theatervorstellungen und Masken in den untergeordneten Städten und in den Dörfern und Landsitzen ihres Großherzogtums für ihre geliebten Untertanen sei nicht nur wegen der Zwietracht und der Streitereien, die aufgrund dieser Vorstellungen unter den Bewohnern entstehen, sondern auch wegen der schlechten Erziehung, der Laster und Skandale, die sich auf diese Weise dort verbreiten, und sie möchte diesen Unannehmlichkeiten wirksam abhelfen. ${ }^{121}$

Bei der Durchsetzung des Erlasses allerdings erwies sich der Monarch einzelnen Einwänden gegenüber nicht immer als unzugänglich ${ }^{22}$. Dennoch: Gefragt waren nun nicht mehr Theatervorstellungen, wie die Burletta in Musica La finta sposa olandese von Marcello da Capua, die die Landbevölkerung in Figline noch 1785 in Eigenregie auf-

20 "IX. In Firenze nel Teatro di Via della Pergola saranno permessi i soliti Veglioni nelle sere del Giovedì, Domenica, e Martedì ultimi del Carnevale; ed in quello degli Intrepidi, o sia della Palla a Corda nelle sere dei due ultimi Lunedì, e penultima Domenica del Carnevale. In tutte le altre Città, e Terre in cui è permesso l'uso dei Veglioni nelle sere del Giovedì, Domenica, Lunedì, e Martedì ultimi del Carnevale. X. Indistintamente poi tutte le Recite che dagl'Impresari, o altri, o per patto, o per altra Causa venivano riservate a profitto, e vantaggio dei Comici, Musici, o Ballerini, o di alcun di loro, chiamate comunemente Beneficiate, resteranno in avvennire soppresse, ed abolite nè mai permesse per verun titolo o pretesto. XI. Saranno proibite generalmente tutte le altre Veglie, e Feste di Ballo a pago, o nomine nelle Case particolari senza la preventiva licenza in Firenze dei Commissari de' Quartieri, e in Provincia dei respettivi Giusdicenti; bene inteso però che tali Licenze non vengano accordate se non a persone di esperimentata probità, e prudenza, e coll'obbligo ingiunto al Capo della Famiglia di rispondere di tutti gli Inconvenienti, che usando della maggior premura avrebbe potuto impedire." (GT 1785, S. 61-62.)

21 "S[ua] A[ltezza] R[eale] sempre vigilante a promuovere il buon costume e all'estirpazione di quei mezzi, che fomentano il vizio, avendo riconosciuto di quanto pregiudizio sia ai Suoi amatissimi Sudditi La Sussistenza dei Teatrali Spettacoli, e delle Maschere nelle Città Subalterne, e nelle Terre, e Castelli del Suo Granducato, non Solo per le dissenzioni, e discordie, che ne derivano da tali Spettacoli fra gli Abitanti, mà ancora per la cattiva educazione, vizi, e scadoli [sic], che vi s'introducono con tali mezzi, e volendo efficacemente riparare ai tali inconvenienti, comanda, e vuole, che in avvenire si osservi costantemente il presente regolamento." (I-Fas: PBG 14, Nr. 185.)

22 Vgl. Chr. Siegert, Cherubini in Florenz, S. 100.

http://www.fzmw.de 
führte $^{23}$. Die Stadt Volterra hatte die Zeichen der Zeit erkannt und erhielt von der semioffiziellen Gazzetta Toscana dafür anerkennende Worte:

"Entsprechend der lobenswerten Sitte, die heutzutage in einige Kollegien Italiens Einzug gehalten hat, die Theatervorstellungen im Karneval durch Vorträge von gelehrten und gefälligen Abhandlungen zu ersetzen, ließen die Rhetorikschüler im Kollegium der Padri delle Scuole Pie in dieser Stadt unter der Leitung von P[ater] Roberto Benazzi am letzten Freitag eine ihrer Akademien hören, die mit einer gut verfaßten Ekloge beschlossen wurde, die auf die Ankunft unseres hervorragenden Königlichen Landvogts anspielte, die Sig. Convittore Canonico Inghiramo Inghirami gedichtet hatte und die von den drei kleinen Herren Convittori Conte Antonio Bentivoglio, Francesco Inghirami und Curzio Incontri kunstvoll und lebhaft vorgetragen wurde. Sie erhielt wie alle anderen Abhandlungen den Applaus des zahlreichen erlesenen Publikums und unseres hochwürdigen Bischofs, der die akademische Veranstaltung mit seiner Anwesenheit beehrte. ${ }^{124}$

Es liegt wohl auf der Hand, daß sich das Publikum bei der beschriebenen Akademie weniger gut unterhielt als bei der Vorstellung der Finta sposa olandese, deren Titel bereits auf Verkleidungs- und Verwechslungsgeschichten mit mehr oder weniger geistreichem Humor hindeutet.

Das Repertoire im historischen Kontext

$$
-6-
$$

Aus dem zitierten Gesetz von 1785 ergibt sich, was im folgenden unter "auf dem Lande" verstanden werden soll: Eben jene kleinen Städte und Gemeinden außer Florenz, Livorno, Pisa und Siena, denen Pietro Leopoldo in den letzten Jahren seiner Regierungszeit die Musiktheaterunterhaltung verbot. Worauf aber mußte die Landbevölke-

23 Vgl. GT 1785, S. 21-22.

24 "A norma del lodevole costume introdotto in oggi in alcuni Collegj d'Italia, di sostituire nel Carnevale alle Teatrali rappresentanze, recite di componimenti eruditi e piacevoli, nel Collegio de' PP. delle Scuole Pie di questa Città, li Scolari della Rettorica sotto la direzione del P[adre] Roberto Benazzi, fecero sentire Venerdì scorso una loro Accademia, che venne chiusa da una ben tessuta Egloga allusiva alla venuta dell'Illustrissimo nostro Sig. Vicario Regio, composta dal Sig. Convittore Canonico Inghiramo Inghirami, che recitata con possesso e brio da' tre piccoli Sigg. Convittori Conte Antonio Bentivoglio, Francesco Inghirami, e Curzio Incontri, riportò con tutte le altre composizioni gli applausi della scelta numerosa udienza e del degnissimo nostro monsig[nore] Vescovo, che onorò di sua presenza l'accademico trattenimento." (GT 1785, S. 31.) 
rung von nun an verzichten? Diesbezüglich sagen die Quellen nur wenig aus. Am ehesten läßt sich zwischen Sprech- und Musiktheater unterscheiden, wobei auch das Sprechtheater kaum ohne musikalische Umrahmung bzw. Untermalung auskam. Im folgenden soll erstens die Frage gestellt werden, was in den Theatern gespielt wurde, und zweitens wann bzw. in welchen Zusammenhängen.

\section{$-7-$}

Wenn in den Quellen Operntitel oder Komponistennamen genannt werden, entsprechen diese meist den bekannten erfolgreichen Buffokomponisten bzw. -opern: Im Karneval 1777 beispielsweise wurde in Pontremoli im Teatro della Rosa "mit allgemeinem Beifall" La sposa fedele von Guglielmi aufgeführt ${ }^{25}$. Am Teatro dell'Accademia degli Affilati in Pescia wurde im Karneval 1762 das "Intermezzo a due voci" L'uccellatrice gegeben, im Sommer 1784 wurde das Dramma giocoso L'italiana in Londra, vermutlich mit der Musik Domenico Cimarosas aufgeführt ${ }^{26}$. In Prato gab es im Frühjahr 1771 La buona figliuola, mutmaßlich mit der Musik von Niccolò Piccinni, und die "Burletta in musica" Il ratto della sposa ${ }^{27}$. In der Karnevalssaison 1775 wurden zwei "burlette in musica", darunter Pasquale Anfossis La finta giardiniera gegeben ${ }^{28}$. Im Sommer des Folgejahres gab es Anfossis Il geloso in cimento sowie L'innocente fortunata von Giovanni Paisiello ${ }^{29}$, dessen Fraschetana ein Jahr später zur Aufführung kam ${ }^{30}$. In welcher Gestalt die Opern gespielt wurden, wird nicht berichtet. Allerdings kann vermutet werden, daß die Bühnenwerke gewöhnlich einschneidende Änderungen und Reduktionen erfuhren. Daß sich bei den Mitwirkenden Wanderungsbewegungen innerhalb der Toskana ausmachen lassen, verwundert nicht. So traten im Frühjahr 1771 in Pistoia Akrobaten auf, die vorher im Florentiner Teatro del Cocomero zu bestaunen waren ${ }^{31}$. In Pisa spielte 1775 eine Compagnia buffa, die aus Livorno $\mathrm{kam}^{32}$. Darüber hinaus bot der lokal bzw. regional begrenzte Raum Auftrittsmöglichkeiten für nicht- oder semiprofessionelle Darstellerinnen und Darsteller. In Fiesole z. B. brachte eine Gruppe von Dilettanten im Teatro dell'Accademia dei Generosi eine erfolgreiche Aufführung von Paisiellos Idolo cinese heraus: "[D]ie Mitwirkenden agieren, obwohl sie Dilettanten

\footnotetext{
25 "[...] con incontro universale" (GT 1777, S. 24).

26 Vgl. E. Garbero Zorzi, L. Zangheri, I teatri storici della Toscana, Bd. VI: Pistoia e provincia, Firenze 1995, S. 330.

GT 1771, S. 67, 83.

GT 1775, S. 3, 11, 23, 26-27, 30.

Vgl. GT 1776, S. 127, 139, 143-144.

Vgl. GT 1777, S. 119.

Vgl. GT 1771, S. 71.

Vgl. GT 1775, S. 107-108.
}

http://www.fzmw.de 
sind, wie professionelle Darsteller, und von professionellen Musikern wird das große Orchester geleitet, das sich aus Dilettanten zusammensetzt. Der Applaus war anhaltend, und einige mußten Arien wiederholen, so gut haben sie sie ausgeführt." ${ }^{\prime 33}$ Die Verantwortlichen hatten darüber hinaus neue Bühnenbilder und Kostüme im chinesischen Stil anfertigen lassen, ${ }^{34}$ so daß Augen und Ohren des Publikums gleichermaßen auf ihre Kosten kamen.

\section{$-8-$}

Die Gesetzgebung zeigte bereits, daß in vielen Provinztheatern die einzige Saison der Karneval war, wobei die Theatergesetze damit eine ohnehin übliche Praxis bestätigten: Generell war der Karneval in Italien die Hauptsaison des Theaterbetriebs. Die Aufführungen begannen in der Provinz häufig später als in den großen Städten, wo die erste Premiere gewöhnlich am zweiten Weihnachtstag stattfand. Auf diese Weise konnten die Impresari eine zweite Produktion sparen. Je kleiner der Ort, desto bescheidener dürften die musiktheatralischen Darbietungen ausgefallen sein. Eine Folge davon war die Bevorzugung kleinerer Gattungen, v. a. des Intermezzos, so daß die Situation im Karneval 1767 in Pistoia, wo ab 21. Januar "varie Tragedie, e Commedie con intermezzi in musica" ${ }^{\prime 35}$ gegeben wurden, wohl als paradigmatisch für das Theaterleben in kleineren Ortschaften gelten kann. Seltener war offenbar der Fall, daß Oper und Ballett kombiniert wurden, wie in derselben Saison aus Arezzo berichtet wird $^{36}$. Üblich war - wie in den italienischen Opernzentren - die Mischung unterschiedlicher Gattungen, die einen unterhaltsamen Theaterabend gewährleistete.

$$
-9-
$$

Charakteristisch ist zudem die Verknüpfung von Theateraufführungen mit den üblichen Feierlichkeiten am Patronatsfest. Auch darauf nahmen die zitierten gesetzlichen Regelungen Rücksicht. In dem Weinort Montepulciano wurde das Fest der Schutzheiligen Agnes als großes Volksfest gefeiert:

"An den ersten drei Tagen dieses Monats wurde das alle drei Jahre stattfindende Fest unserer Schutzheiligen, der Hl. Agnes, mit feierlichem Pomp begangen. Die

"[G]li Attori, benchè dilettanti operano da veri Professori come da Professori, è regolata la numerosa Orchestra composta di Dilettanti. Fu continovo l'applauso, e convenne ad alcuni ripetere delle arie, tanto erano bene eseguite." (GT 1775, S. 168.)

Vgl. ebd.

GT 1767, S. 23.

Vgl. GT 1767, S. 27. Zur Repertoirezusammensetzung vgl. auch die Tabelle im Anhang. 
erlesene Musik, die von verschiedenen Untertanen mit der größten Kunstfertigkeit dargeboten wurde, das Feuerwerk, die beiden Pferderennen [...] und insbesondere die mit den von Signor Gucci choreographierten Balletten geschmückte Burletta in musica mit dem Titel la Giardiniera, in der sich Sig. Maddalena Granati auszeichnete, die mit ihren jungen Jahren zu großen Erwartungen berechtigt; alles hat dazu beigetragen, die große Zahl von Fremden zu unterhalten, die zu diesem Anlaß hier hergekommen sind. ${ }^{137}$

Solche Vorstellungen konnten deutlich opulenter ausfallen als die Karnevalsvorstellungen, da die Theater außerhalb des regulären Betriebs nicht mit den repräsentativen Häusern konkurrierten ${ }^{38}$. Gleichzeitig bedeuteten derartige Engagements einen willkommenen Zusatzverdienst für die auftretenden Künstler. Schließlich profitierten die Landgemeinden und ihre Theater von der Gewohnheit der Villeggiatura, die ein Teil der wohlhabenden Stadtbevölkerung - potentielle zahlungskräftige Theaterbesucher - auf dem Lande verbrachte. Ganz besondere Besucher waren der Monarch bzw. Mitglieder der Herrscherfamilie oder Staatsgäste. Von der Aufführung der Buona figliuola in Prato 1771 berichtete die Gazzetta Toscana vielleicht nur aus dem Grund, weil sie in Anwesenheit des Großherzogs gespielt wurde:

"Sonntag abend ungefähr um 6 Uhr kam hier mit einem Gespann Seine Königliche Hoheit unser Herrscher aus der Königlichen Villa in Poggio a Caiano an, der nach einer kurzen Stadtrundfahrt am Palazzo Pretorio ausstieg, wo er von unserem Sig. Commissario empfangen wurde, und nach einem kurzen Aufenthalt begab er sich in Begleitung des Kaiserlichen Ministers Conte di Wildseck und seines Gefolges ins öffentliche Theater, um die Burletta in musica La buona figliuola zu hören, die sowohl dank der Musik als auch wegen des Könnens der Akteure den Beifall Seiner Königlichen Hoheit erhielt, die, nachdem sie die

37 "Nei primi tre giorni del corrente, fu sollennizzata con solenne pompa la festa triennale della nostra Protettrice S. Agnese. La scelta musica eseguita da vari soggetti della più nota abilità, $i$ fuochi d'artifizio, le due corse, di cavalli [...] e sopra tutto la Burletta in musica intitolata la Giardiniera dove si distingue la Sig. Maddalena Granati, che da di se stessa in fresca età grande espettativa, decorata dai balli d'invenzione del Signor Gucci, tutto ha contribuito a divertire la gran quantità di Forestieri, che in tale occasione quà sono concorsi." (GT 1776, S. 79-80; bei der erwähnten Oper handelt es sich möglicherweise um Anfossis Finta giardiniera.) Vgl. auch GT 1767, S. 124.

http://www.fzmw.de 
Räume des öffentlichen Stadthauses, das dem Theater angeschlossen ist, besichtigt hatte, sofort wieder nach Poggio zurückkehrte. ${ }^{139}$

Aus einer weiteren Notiz kann man entnehmen, daß diese "Burletta", ebenso wie eine zweite, gewöhnlich an Feiertagen aufgeführt wurde ${ }^{40}$.

$$
-10-
$$

Einen Sonderfall bildet das Theater der in dem Zitat erwähnten großherzoglichen Villa in Poggio a Caiano, wo der Monarch mit seiner Familie gewöhnlich seine Landaufenthalte verbrachte. In den Jahren 1771 bis 1773 wurden dort Opere buffe produziert; danach spielten die Comici francesi während der Villeggiature ${ }^{41}$. Aufgeführt wurden im Herbst 1771 Il finto pazzo per amore von Antonio Sacchini, L'isola d'amore und Le finte gemelle, im Frühjahr des folgenden Jahres Il barone di rocca antica und Niccolò Piccinnis La pescatrice. Diese Oper wurde im Herbst desselben Jahres wieder aufgenommen und durch Piccinnis Americano und Pietro Alessandro Guglielmis Cacciatori ergänzt; im Herbst 1773 schließlich gab es die Florentiner Produktion Amor per rigiro mit Musik von Giovanni Marco Rutini auf einen Text Abate Niccolò Tassis, $I$ rovinati von Florian Gassmann und La reconciliazione del villaggio ${ }^{42}$. Von den genannten Bühnenwerken gibt es nur für L'isola d'amore, Le finte gemelle und La reconciliazione keine Librettodrucke. $\mathrm{Zu}$ den übrigen Opern ließ der Großherzog zwischen 150 und 300 Libretti anfertigen, von denen einige auf Papier mit Goldschnitt gedruckt und in einen Seidenumschlag eingebunden wurden. Das Orchester war mit Florentiner Hofmusikern besetzt ${ }^{43}$. Dieser repräsentative Aufwand macht die Bandbreite des ländlichen toskanischen Opernlebens besonders sinnfällig.

"Domenica scorsa circa le ore 6. della sera pervenne qui in muta dalla Real Villa del Poggio a Caiano S[ua] A[ltezza] R[eale] nostro Signore, quale dopo aver fatto un breve giro per questa Città smontò al palazzo Pretorio, ove fu ricevuto da questo sig. Commissario, e dopo breve dimora si portò al pubblico Teatro in compagnia del sig. Conte di Wildseck Ministro Cesareo, e della sua Nobil Comitiva, a sentire la Burletta in musica intitolata la Buona Figliuola, che sì per la musica che per l'abilità degli attori incontrò il gradimento della R[eale] A[ltezza] S[ua] la quala [sic] dopo di avere vedute le stanze del pubblico palazzo annesse a detto Teatro, immediatamente riprese il cammino alla volta del Poggio." (GT 1771, S. 67.) Vgl. GT 1771, S. 83.

41 Vgl. Litta Medri, Un episodio culturale nella Villa Medicea di Poggio a Caiano: il Teatro di Corte al Tempo di Pietro Leopoldo, in: Zeffiro Ciuffoletti, Leonardo Rombai (Hg.), La Toscana dei Lorena. Riforme, territorio, società, Atti del Convegno di studi (Grosseto, 27-29 novembre 1987), (Biblioteca storica toscana, Sezione di Storia del Risorgimento, Bd. 19) Firenze 1989, S. 379-390, bes. S. 389.

42 Vgl. I-Fas: Imperiale e Reale Corte [im folgenden abgekürzt $I R C$ ] 229, Nr. 60; 230, Nr. 30, 66; 231, Nr. 45.

43 Vgl. I-Fas: IRC 229, Nr. 60; 230, Nr. 30, 66; 231, Nr. 45.

FZMw Jg. 7 (2004) 
Ausblick

$-11-$

Bis in die 1780er Jahre hinein ergibt sich aus Zeitungsberichten und Archivmaterial eine lebendige Musiktheatertradition auch außerhalb der städtischen Zentren der Toskana, die Ansatzpunkte für weitere Forschungen bietet. Dokumentierte Theaterbauten, nachgewiesene Aktivitäten von Impresari sowie nicht weiter spezifizierte Aufführungshinweise in der Presse werfen Fragen nach den gespielten Bühnenwerken und ihren Bearbeitungen, den Darstellern - gerade auch, wenn es sich um Laien handelt -, ihrer Bezahlung usw. auf. Anhand der Wanderungen von Theatertruppen und einzelnen Darstellern könnten Rezeptionswege nachvollzogen werden. Bereits zum jetzigen Zeitpunkt ist festzustellen, daß die Oper im 18. Jahrhundert ungeachtet aller Gesetze weiten Teilen der Bevölkerung zumindest zeitweise verfügbar war. Sie war wesentlich und insbesondere in der Provinz Teil einer umfassenden Festkultur, sei es im Rahmen des Karnevals, eines Heiligenfestes oder eines Staatsbesuchs. Damit ergibt sich eine entscheidende Differenz im Vergleich zu einem Medium des 20. Jahrhunderts, der sich in vieler Hinsicht aufdrängt: dem Film, der - so Anselm Gerhard - die Oper "zu einem guten Teil beerbt hat. ${ }^{\prime 44}$ Erinnert sei vor allem an die dramaturgischen Stereotypen, auf die sich Opern- bzw. Filmhandlungen zurückführen lassen, normierte Rollen und typisierte Szenen wie Verkleidungs- und Verwechslungsszenen. Zudem übernahm das neue Medium die Lokalitäten: Mehrere Theater des 18. Jahrhunderts, z. B. das Teatro dell'Accademia dei Rinnovati Concordi in Monte San Savino oder das Regio Teatro in Fucecchio, wurden im 20. Jahrhundert in Kinos umfunktioniert ${ }^{45}$. Doch der Film war und ist Teil einer allseits verfügbaren Alltagskultur. Die Oper hingegen ist vermutlich nie Bestandteil des täglichen Lebens geworden.

44 Anselm Gerhard, Die Verstädterung der Oper. Paris und das Musiktheater des 19. Jahrhunderts, Stuttgart 1992, S. 5. Vgl. auch Reinhard Strohm, Die italienische Oper im 18. Jahrhundert, (Taschenbücher zur Musikwissenschaft, hg. von Richard Schaal, Bd. 25) Wilhelmshaven 1979, S. 11-12.

45 Vgl. E. Garbero Zorzi, L. Zangheri, I teatri storici della Toscana, Bd. IV, S. 279; Bd. VII: Provincia di Firenze. Prato e provincia, Firenze 1998, S. 358.

http://www.fzmw.de 


\section{Anhang}

$-12-$

Theater in der Toskana außerhalb der Städte Florenz, Siena, Pisa und Livorno in den Jahren 1767, 1775-1776 und 1782-1784 nach der Gazzetta Toscana:

\begin{tabular}{|c|c|c|c|c|}
\hline Zeit & Ort & Theater & Repertoire & Quelle \\
\hline $\begin{array}{l}\text { Karneval } 1767(\mathrm{ab} \\
\text { 21.1.) }\end{array}$ & Pistoia & $\begin{array}{l}\text { Pubblico } \\
\text { Teatro }\end{array}$ & $\begin{array}{l}\text { "varie Tragedie, e Commedie } \\
\text { con intermezzi in musica" }\end{array}$ & GT 1767, S. 23 \\
\hline $\begin{array}{l}\text { Ende Jan./Anfang } \\
\text { Febr. } 1767 \\
\end{array}$ & Arezzo & Teatro & $\begin{array}{l}\text { "l'Opera intitolata Sesostri, } \\
\text { con balli" }\end{array}$ & GT 1767, S. 27 \\
\hline 28.2 .1767 & Prato & Teatro & $\begin{array}{l}\text { Farsa zur Huldigung des } \\
\text { Großherzogs und Konzert }\end{array}$ & GT 1767, S. 44 \\
\hline $\begin{array}{l}\text { Ende Febr./Anfang } \\
\text { März } 1767\end{array}$ & Volterra & Teatro privato & Scipione Maffei: Merope & GT 1767, S. 47 \\
\hline $\begin{array}{l}\text { 22.7. bis Ende Aug. } \\
1767\end{array}$ & Pistoia & \begin{tabular}{|l|} 
Pubblico \\
Teatro
\end{tabular} & Burletta in musica und Ballett & \begin{tabular}{|l|} 
GT 1767 \\
S. 124,128 \\
144,148 \\
\end{tabular} \\
\hline Karneval 1775 & Arezzo & $\begin{array}{l}\text { Pubblico } \\
\text { Teatro }\end{array}$ & $\begin{array}{l}\text { Opere serie (Il gran Cidde, Il } \\
\text { Creso) und Ballett; } \\
\text { Commedie }\end{array}$ & $\begin{array}{l}\text { GT 1775, S. } 7, \\
15\end{array}$ \\
\hline Karneval 1775 & Bibbiena & Teatro & $\begin{array}{l}\text { "commedia intitolata 'l'Erede } \\
\text { Fortunata'" und "la Tragedia } \\
\text { intitolata 'L'Alzira [...] } \\
\text { framezzata da una curiosa, e } \\
\text { vaga farsa nominata la } \\
\text { Maestra Scaltra" } \\
\end{array}$ & GT 1775, S. 39 \\
\hline Karneval 1775 & Cortona & $\begin{array}{l}\text { "nuovo Teatro } \\
\text { de i nobili sigg. } \\
\text { Uniti" } \\
\end{array}$ & \begin{tabular}{|l|} 
Tamas Culicam und eine \\
weitere Opera seria mit 2 \\
Abbattimenti
\end{tabular} & GT 1775, S. 40 \\
\hline Karneval 1775 & Prato & \begin{tabular}{|l|} 
Pubblico \\
Teatro
\end{tabular} & $\begin{array}{l}\text { Pasquale Anfossi: La finta } \\
\text { giardiniera und eine weitere } \\
\text { Burletta in musica }\end{array}$ & \begin{tabular}{|l}
$G T 1775$, S. 3 \\
$11,23,26-27$ \\
30
\end{tabular} \\
\hline Karneval 1775 & Prato & \begin{tabular}{|l|} 
Teatro del Real \\
Collegio \\
Cicognini \\
\end{tabular} & $\begin{array}{l}\text { "varie Opere Comiche, e } \\
\text { Tragiche" }\end{array}$ & GT 1775, S. 30 \\
\hline Mitte Febr. 1775 & Fivizano & \begin{tabular}{|l|} 
Pubblico \\
Teatro \\
\end{tabular} & $\begin{array}{l}\text { "due opere intitolate la Semi- } \\
\text { ramide, e la Donna Sola" }\end{array}$ & GT 1775, S. 23 \\
\hline ab 14.2.1775 & Pistoia & Seminario & $\begin{array}{l}\text { "rappresentanze tragiche, e } \\
\text { comiche }[\ldots] \text { tramezzate da } \\
\text { balli" }\end{array}$ & $\begin{array}{l}\text { GT 1775, S. 27, } \\
34,39\end{array}$ \\
\hline $\begin{array}{l}\text { Herbst } 1775 \text { (bis } \\
\text { Anfang Nov.) }\end{array}$ & S. Sepolcro & & Schauspiel & \begin{tabular}{|l|}
$G T 1775$, \\
S. 164,175 \\
\end{tabular} \\
\hline 18.10 .1775 & Fiesole & \begin{tabular}{|l|} 
Teatro \\
dell'Accademia \\
dei Generosi \\
\end{tabular} & $\begin{array}{l}\text { Giovanni Paisiello: L'idolo } \\
\text { cinese }\end{array}$ & $\begin{array}{l}G T 1775, \\
\text { S. } 167-168,175\end{array}$ \\
\hline Karneval 1776 & Cortona & \begin{tabular}{|l|} 
"Teatro grande \\
di questa \\
Nobiltà" \\
\end{tabular} & $\begin{array}{l}\text { "diverse sceltissime } \\
\text { Commedie" }\end{array}$ & GT 1776, S. 35 \\
\hline $\begin{array}{l}1 .-3.5 .1776 \\
\text { (S. Agnese) }\end{array}$ & Montepulciano & & $\begin{array}{l}\text { Burletta in musica } L a \\
\text { giardinera und Ballett }\end{array}$ & GT 1776, S. 80 \\
\hline $\begin{array}{l}\text { ab 24.7.1776 } \\
\text { (S. Jacopo) }\end{array}$ & Pistoia & Teatro & $\begin{array}{l}\text { "Dramma intitolato l'Isola } \\
\text { d'Amore" }\end{array}$ & \begin{tabular}{|l|}
$G T 1776$, \\
S. 124,139 \\
\end{tabular} \\
\hline ab 22.8.1776 & Pistoia & & $\begin{array}{l}\text { Marco Rutini: Il finto amante } \\
\text { und Ballett }\end{array}$ & GT 1776, S. 139 \\
\hline $1 .-25.8 .1776$ & Prato & \begin{tabular}{|l|} 
Pubblico \\
Teatro \\
\end{tabular} & $\begin{array}{l}\text { Pasquale Anfossi: Il geloso in } \\
\text { cimento }\end{array}$ & GT 1776, S. 127 \\
\hline
\end{tabular}




\begin{tabular}{|c|c|c|c|c|}
\hline 30.8.-11.9.1776 & Prato & $\begin{array}{l}\text { Pubblico } \\
\text { Teatro }\end{array}$ & $\begin{array}{l}\text { Giovanni Paisiello: } \\
\text { L'innocente fortunata }\end{array}$ & $\begin{array}{l}\text { GT 1776, } \\
\text { S. } 139,143-144, \\
148\end{array}$ \\
\hline 29.9.-7.11.1776 & Torrita & $\begin{array}{l}\text { Teatro } \\
\text { dell'Accademia }\end{array}$ & $\begin{array}{l}\text { Voltaire: Zaira, Komödie } \\
\text { Goldonis, Niccolò Piccinni: I } \\
\text { servi astuti }\end{array}$ & GT 1776, S. 163 \\
\hline Karneval 1782 & S. Fiora & & $\begin{array}{l}\text { Carlo Goldoni: "le due } \\
\text { Pamele fanciulla e maritata" } \\
\text { und Filosseno Olitense: Il } \\
\text { Tutore Politico, o sia } \\
\text { l'Amante in Delirio } \\
\text { (Intermezzo) }\end{array}$ & GT 1782, S. 27 \\
\hline ab 6.1.1782 & Poggibonsi & $\begin{array}{l}\text { "nuovo Teatro } \\
\text { detto dei } \\
\text { Costanti" }\end{array}$ & $\begin{array}{l}\text { "Dramma la Didone } \\
\text { Abbandonata" und Giuseppe } \\
\text { Brunetti: Smargiasso pauroso } \\
\text { (Farsa) }\end{array}$ & GT 1782, S. 8 \\
\hline Karneval 1783 & Montepulciano & Teatro & $\begin{array}{l}\text { Drammi buffi per musica } L a \\
\text { Contadina in Corte und } \\
\text { L'Italiana in Londra }\end{array}$ & $\begin{array}{l}\text { GT 1783, S. 15, } \\
34\end{array}$ \\
\hline Karneval 1783 & Pontadera & Nuovo Teatro & $\begin{array}{l}\text { Pietro Metastasio: "diverse } \\
\text { Opere", Abate Ringhieri: } \\
\text { Adelasia in Italia (Tragödie), } \\
\text { Olimpiade (Opera seria) } \\
\end{array}$ & GT 1783, S. 31 \\
\hline $\begin{array}{l}\text { Ende Juli } 1783 \\
\text { (S. Jacopo) }\end{array}$ & Pistoia & Teatro & $\begin{array}{l}\text { Dramma giocoso La locanda } \\
\text { und Ballett }\end{array}$ & GT 1783, S. 123 \\
\hline Karneval 1784 & Gambassi & Teatro & $\begin{array}{l}\text { Werke Metastasios und Farse } \\
\text { buffe }\end{array}$ & GT 1784, S. 31 \\
\hline Frühjahr 1784 & $\begin{array}{l}\text { Castelnuovo in } \\
\text { Garfagnana }\end{array}$ & $\begin{array}{l}\text { Nuovo Teatro } \\
\text { dei sei } \\
\text { Associati }\end{array}$ & $\begin{array}{l}\text { Auftritte der Schauspieler- } \\
\text { gesellschaft Santini }\end{array}$ & GT 1784, S. 111 \\
\hline Sommer 1784 & Barga & Teatro & $\begin{array}{l}\text { Auftritte der Schauspieler- } \\
\text { gesellschaft Santini }\end{array}$ & GT 1784, S. 111 \\
\hline ab 8.8.1784 & Pistoia & & $\begin{array}{l}\text { L'italiana in Londra (Opera } \\
\text { buffa) }\end{array}$ & GT 1784, S. 130 \\
\hline
\end{tabular}

